UMD-PP-95-61

November, 1994

\title{
Almost Degenerate Neutrinos with Maximal Mixing
}

\author{
R.N. Mohapatra円 and S. Nussinov \\ Department of Physics and Astronomy, University of Maryland, College Park, \\ $M D 20742$
}

\begin{abstract}
We point out that if the observed deficit of solar and atmospheric neutrinos are to be understood as consequences of oscillations between different neutrino flavors, the simplest way to reconcile it with mixed dark matter picture of the universe and a possible sub-electron volt upper limit on the $\nu_{e}$ Majorana mass is to have a scenario of three light almost degenerate Majorana neutrinos $\left(\nu_{e}, \nu_{\mu}\right.$ and $\left.\nu_{\tau}\right)$ with maximal CP-violating mixing among among all three of them. We discuss theoretical scenarios which may lead to such a mass and mixing pattern. We also discuss a scenario where two of the three degenerate neutrinos are maximally mixed.
\end{abstract}

\footnotetext{
${ }^{1}$ Work supported by the National Science Foundation Grant PHY-9119745

${ }^{2}$ Permanent address: Department of Physics and Astronomy, Tel Aviv University, Tel Aviv, Israel.
} 
I. The conventional hierarchical "see-saw" mechanism[1] for neutrino masses and mixings provides an overall view of neutrino physics which is quite attractive. In this approach, the left-handed neutrinos are Majorana particles and their masses are given by:

$$
m_{\nu_{i}^{L}} \simeq\left(m_{\nu_{i}}^{D}\right)^{2} / M_{N_{R}}
$$

where the $m_{\nu_{i}}^{D}$ is the Dirac mass connecting the active left-handed flavor of the neutrino $\nu_{i L}$ with the sterile right-handed one denoted by $N_{i R}$ and $M_{N_{R}}$ is the Majorana mass for the heavy sterile right-handed neutrino. The formula in equation (1) is obtained by diagonalizing a generic $\nu_{L}-N_{R}$ mass matrix of the form:

$$
M=\left(\begin{array}{cc}
0 & m_{\nu}^{D} \\
m_{\nu}^{D^{T}} & M_{N_{R}}
\end{array}\right)
$$

Let us note the particular feature of this matrix that the $\nu_{L} \nu_{L}$ entry is assumed to vanish. The light neutrino masses then exhibit the hierarchical mass pattern if the Dirac masses are assumed to be related to the charged fermion masses of the corresponding generation and no strong hieararchy is assumed to exist among the right-handed neutrino masses. In grand unified theories such as $\mathrm{SO}(10)$ or $E_{6}$, such relationsbetween the Dirac masses of neutrinos and the charged fermion masses do indeed arise. Likewise one expects also a hierarchical pattern in the mixings between the different generations. Detailed predictive grand unified models based on the $S O(10)$ group do indeed confirm these intuitive expectations for the hierarchical pattern. As far as the absolute values of the masses is concerned, it is clearly determined by the magnitude of $M_{N_{R}}$ which is proportinal to the breaking of the local $B-L$ symmetry in the theory. In the framework of grand unified theories such as $\mathrm{SO}(10)$, the present LEP data completely determine the value of the $B-L$ symmetry breaking scale, $M_{B L}$. For non-supersymmetric models [2], this scale is anywhere between $10^{10} \mathrm{GeV}$ to $10^{13.5} \mathrm{GeV}$ and for the supersymmetric case, it is around $10^{15}$ to $10^{16} \mathrm{GeV}$. The conventional see-saw formula then implies that the value of the electron neutrino mass is in the micro-eV range, that of muon neutrino is in the milli-eV range and that of the tau neutrino is in the $\mathrm{eV}$ range. This kind of neutrino spectrum has the following experimental implications; (i) it can account for the hot dark matter (HDM) of the universe if $m_{\nu_{\tau}} \simeq 5-7 \mathrm{eV}$; (ii) it can explain all four solar neutrino experiments using the attractive MSW mechanism for $\nu_{e}-\nu_{\mu}$ oscillation for $m_{\nu_{\mu}} \simeq 10^{-3} \mathrm{eV}$ as expected from these models. However given a scenario that explains the solar neutrino puzzle and HDM of the universe, it cannot explain the deficit of atmospheric muon neutrinos. This picture can be confirmed by the proposed CHORUS[3] and NOMAD[3], implying some new mechanism to understand the atmospheric neutrino deficit other than neutrino oscillations. 
It was proposed in ref. [4], that the simplest way to accomodate the solar neutrino puzzle, the atmospheric neutrino puzzle and the MDM picture of the universe is to assume that the three known neutrinos are almost degenerate with a common mass of around $2 \mathrm{eV}$. The reason for near degeneracy is that the mass difference between $\nu_{\mu}$ and $\nu_{\tau}$ required to solve the atmospheric neutrino deficit is [5] of order $5 \times 10^{-3}$ to $.5 \mathrm{eV}^{2}$. So if we want a $\nu_{\tau}$ with a mass in the few $\mathrm{eV}$ range to become the hot dark matter, $m_{\nu_{\mu}}$ must be in the $\mathrm{eV}$ range and be almost degenerate with the $\nu_{\tau}$. Now since all solutions of the solar neutrino puzzle using neutrino oscillations also require that the $\nu_{e}-\nu_{\mu}$ mass difference must be very small (i.e. $10^{-5} \mathrm{eV}$ in the case of MSW solution or $10^{-10} \mathrm{eV}^{2}$ in the case of vacuum oscillation), we must have $\nu_{e}$ and $\nu_{\mu}$ nearly degenerate. A complete understanding of all three puzzles therefore require that the three light neutrinos must be degenerate with masses around $2 \mathrm{eV}$ or so.

Theoretical appeal for such models owes its origin to an early observation 6] that in many models that implement the see-saw mechanism, the actual seesaw mass matrix has the form:

$$
M=\left(\begin{array}{cc}
m_{L L} & m_{\nu}^{D} \\
m_{\nu}^{D^{T}} & M_{N_{R}}
\end{array}\right)
$$

where $m_{L L}=f \frac{v_{W}{ }^{2}}{V_{B L}}$ and $M_{N_{R}}=f v_{B L}$. The coefficient $f$ is matrix in generation space, which is not expected to have a strong dependence on the generations. Note that $m_{L L}$ leads to a direct mass for the light neutrinos which while being of seesaw type (i.e. inversely proportional to $v_{B L}$, can be assumed to be independent of generations and will not therefore have the hierarchical pattern given in eq.(1). The correct pattern of neutrino masses given by the the eq.(3) is then:

$$
m_{\nu_{i}} \simeq f_{i} \frac{v_{W}^{2}}{V_{B L}}-\frac{m_{\nu_{i}}^{D^{2}}}{f_{i} v_{B L}}
$$

Note that if all $f_{i}$ 's are set to be equal ( perhaps by some symmetry [7] ), then we have the desired almost degenerate scenario .

An unavoidable prediction of these models is that $m_{\nu_{e}} \simeq 2 \mathrm{eV}$ or so as mentioned. Since in the usual see-saw picture, the neutrinos are Majorana fermions, the present generation of neutrinoless double beta decay experiments 8] should observe a signal at the appropriate level. It appears that the Heidelberg-Moscow double beta decay experiment has now an upper limit on the $\nu_{e}$ mass $.68 \mathrm{eV}[9]$ at the $90 \%$ confidence level. A question that now arises is whether the almost degenerate neutrino mass scenario can be maintained while not being inconsistent with the sub-eV 
mass limits on $\nu_{e}$ suggested in experiments. One could perhaps assume that there are uncertainties in nuclear matrix elements which allow the degenerate scenario to remain viable. There are however recent cosmological observations indicating a higher value of Hubble constant ( $h \simeq .80$ in units of $100 \mathrm{~km} / \mathrm{sec} / \mathrm{Mpc}$ ) which taken seriously, would imply that a $30 \%$ hot dark matter content in the degenerate neutrino scenario would require common mass $m_{\nu_{i}} \simeq 6 \mathrm{eV}$. It would certainly be hard to reconcile such a mass value with the $\beta \beta_{0 \nu}$ results.

In this letter, we suggest that a way to reconcile the possible sub-eV $\beta \beta_{0 \nu}$ upper limit on $m_{\nu_{e}}$ with the almost degenerate neutrino spectrum is to assume that the light neutrinos have a maximal CP-violating mixing among themselves [10, 11]. This may help to reconcile qualitatively all known data for the neutrinos. This mixing pattern is a generalization of the symmetric maximal mixing scheme proposed in 10 in order to understand the reduction of the neutrino flux in the chlorine experiment. We also note an alternative scenario where one has a maximal mixing between $\nu_{e}$ and one of the other neutrinos .

\section{Sub-eV effective $\nu_{e}$ mass and maximally mixed neutrinos:}

The starting point of the present paper is the well known result that in neutrinoless double beta decay, one measures the effective mass of the light neutrino $\left\langle m_{\nu_{e}}\right\rangle \equiv \Sigma_{i} U_{e i}^{2} m_{\nu_{i}}$ and therefore $\beta \beta_{0 \nu}$ amplitude is not only sensitive to the absolute values of the neutrino mass but also to the their mixing pattern. It is then clear that the simplest way to reconcile the almost degenerate scenario for neutrinos with a sub-eV upper limit on the effective Majorana mass of $\nu_{e}$ as given by neutrinoless double beta decay experiments is that the neutrino mixing matrix be maximal and CP-violating among all three or between any two generations one of which is the $\nu_{e}$. We will discuss both these possibilities below separately. In what follows, we define the neutrino mixing matrix as follows: $L_{w k}=\frac{g}{2 \sqrt{2}} W^{-} \bar{e}_{i L} U_{\nu i j} \nu_{j L}+$ h.c. where we have omitted the Lorentz vector character of the interaction.

\section{Case A: Maximal mixing among three generations:}

The form of the mixing matrix in this case is given by:

$$
U_{\nu}=\frac{1}{\sqrt{3}}\left(\begin{array}{ccc}
1 & \omega & \omega^{2} \\
1 & \omega^{2} & \omega \\
1 & 1 & 1
\end{array}\right)
$$

where $\omega=e^{\frac{2 \pi i}{3}}$. Note that any redefinition of fields to remove these phases makes 
the Majorana masses complex and leads to the same result. If we write the neutrino masses to be $m_{\nu_{e}}=m_{0}+\delta_{e}, m_{\nu_{\mu}}=m_{0}+\delta_{\mu}$ and $m_{\nu_{\tau}}=m_{0}+\delta_{\tau}$ with $\delta_{e}, \delta_{\mu}, \delta_{\tau} \ll$ $m_{0} \simeq$ few $\mathrm{eV}$, the effective mass measured in the $\beta \beta_{0 \nu}$ experiment is given by $\left\langle m_{\nu_{e}}\right\rangle \equiv \Sigma_{i=\nu_{e}, \nu_{\mu}, \nu \tau} U_{e i}^{2} m_{i}=\delta_{e}+\omega^{2} \delta_{\mu}+\omega \delta_{\tau}$, ( which follows from the fact that $\left.1+\omega+\omega^{2}=0\right)$ which is clearly in the sub-eV range. In fact, the solar and atmospheric neutrino data imply that $\delta_{e}, \delta_{\mu} \ll \delta_{\tau} \leq .25 \mathrm{eV}$ (or .08 eV) if we assume, $m_{0}=2 \mathrm{eV}$ ( or $6 \mathrm{eV}$ ). Thus, if the atmospheric neutrino data becomes precise enough to fix the $\Delta_{\mu-\tau}^{2}$, the $\beta \beta_{0 \nu}$ decay experiment can be used to test this hypothesis since both the Heidelberg-Moscow ${ }^{76} \mathrm{Ge}$ experiment and the NEMO 3 experiment using ${ }^{100} \mathrm{Mo}$ [12 can probe effective Majorana $\nu_{e}$ mass $\left\langle m_{\nu_{e}}\right\rangle$ down to $.1 \mathrm{eV}$.

Let us now briefly address the question of how one understands the solar and atmospheric neutrino data in this picture. The popular MSW explanation of the solar neutrino puzzle is not applicable in this case due to large values of the mixing angles [13]. There are however two other possible approaches using vacuum oscillation. It was noted sometime back 10 that if the $\nu_{e}-\nu_{\mu}$ mass difference square is much larger than $10^{-10} \mathrm{eV}^{2}$, then the solar $\nu_{e}$ flux gets suppressed by a factor $1 / 3$. While this is perfect for understanding the Chlorine data, its predictions for the Kamiokande and Gallium data[14] are too small compared to observations. The other possibility has been discussed in a recent paper by Kim and Lee 15 where one makes the choice of mass differences $\Delta m_{e \mu}^{2} \simeq 10^{-10} \mathrm{eV}^{2}$ and $\Delta m_{\mu-\tau}^{2} \simeq$ $10^{-1}-10^{-3} \mathrm{eV}^{2}$. As emphasized in ref 15], in the case of the atmospheric neutrinos, one has both the $\nu_{e}-\nu_{\mu}$ as well as $\nu_{\mu}-\nu_{\tau}$ oscillation operative and one can fit the atmospheric neutrino data. As far as the solar neutrino data is concerned, it is not easy to fit all data simultaneously 15]; however, allowing for two standard deviations in the gallium data, a fit has been obtained in ref.[15]. It therefore follows that if present data in all solar neutrino experiments are confirmed by future experiments, this maximal mixing scenario will be ruled out. At the present time, however, entertaining such a possibility is perhaps reasonable.

\section{Case B: Maximal mixing between $\nu_{e}$ and $\nu_{\tau}$ :}

There are two possibilities for mixing in this case: either between $\nu_{e}-\nu_{\mu}$ or between $\nu_{e}$ and $\nu_{\tau}$. Let us illustrate this using the second case. The neutrino mixing matrix then takes the following form:

$$
U_{\nu}=\left(\begin{array}{ccc}
\frac{1}{\sqrt{2}} & \frac{\beta}{\sqrt{2}} & \frac{i}{\sqrt{2}} \\
-\beta c+\frac{i s}{\sqrt{2}} & c+\frac{i s \beta}{\sqrt{2}} & \frac{s}{\sqrt{2}} \\
\beta s+\frac{i c}{\sqrt{2}} & -s+\frac{i \beta c}{\sqrt{2}} & \frac{c}{\sqrt{2}}
\end{array}\right)
$$


We assume that $\beta \ll 1$ and $c$ and $s$ stand for the sine and cosine of an angle which will be fixed by the atmospheric and solar neutrino data. To see if this pattern is viable, let us define $\Delta_{i j}=\left|m_{\nu_{i}}^{2}-m_{\nu_{j}}^{2}\right|$ and $y_{i j}(L) \equiv \sin ^{2}\left(\frac{\Delta_{i j} L}{4 E}\right)$. There are then two cases: (i): $\Delta_{12} \ll \Delta_{13} \simeq \Delta_{23}$, which corresponds to $\delta_{e}, \delta_{\mu} \ll \delta_{\tau}$; (ii): $\Delta_{13} \ll \Delta_{12} \simeq \Delta_{32}$ corresponding to $\delta_{e}, \delta_{\tau} \ll \delta_{\mu}$.

In case (i), the survival probability for the solar neutrino in the vacuum oscillation approximation, is given by:

$$
P\left(\nu_{e} \rightarrow \nu_{e}\right) \simeq 1-y_{13}\left(L_{S}\right)
$$

where $L_{S}$ denotes the Earth-Sun distance. For the atmospheric neutrinos, we get in the simple approximation where we ignore the effects of flux and effects at the detector,

$$
\frac{N_{\mu}}{N_{e}} \simeq 2 \frac{1-\left(c^{2} s^{2}+.5 s^{2}\right) y_{13}\left(L_{A}\right)}{1-\left(1-2 s^{2}\right) y_{13}\left(L_{A}\right)}
$$

where $L_{A}$ denotes the typical distance travelled by the atmospheric neutrinos ( of the order of $10^{3}$ to $10^{4} \mathrm{Km}$.). It is then clear that for $y_{13}\left(L_{A}\right) \approx 1$, there exists a range of values for the free mixing parameter $s$ for which one can fit the atmospheric neutrino observations. However, for these parameters, eq.(7a) for the case of solar neutrinos gives a prediction that solar neutrino flux is energy independent and is reduced by $50 \%$ in all experiments ( since $y_{13} \simeq .5$ ) which is in disagreement with observations.

Turning now to case (ii), we get for the reduction in solar neutrino flux,

$$
P\left(\nu_{e} \rightarrow \nu_{e}\right) \simeq 1-y_{13}\left(L_{S}\right)
$$

In order to understand the solar neutrino data, we would like $y_{13}\left(L_{S}\right)$ to be of order one, which requires $\Delta_{13} \simeq 10^{-10} \mathrm{eV}^{2}$. A careful analysis [16] in this case leads to the conclusion that while it possible to obtain a fit to the present data, it is not a very good fit, though it cannot be definitely excluded. Using the fact that $y_{13}\left(L_{S}\right) \simeq 1$ implies $y_{13}\left(L_{A}\right) \ll 1$, for the case of atmospheric neutrinos, we find :

$$
\frac{N_{\mu}}{N_{e}} \simeq 2\left(1-4 c^{2} s^{2} y_{12}\left(L_{A}\right)\right)
$$

As far as the atmospheric neutrino data is concerned, one can obtain a good fit assuming $\Delta_{12} \simeq 10^{-2} \mathrm{eV}^{2}$. So unlike the case (i) described above, case (ii) may be acceptable as a viable scenario until the solar neutrino data becomes more definitive. 
In summary, we feel that two of the scenarios outlined above may provide a reasonable description of all existing observations on neutrinos ( i.e. solar, atmospheric, $\beta \beta_{0 \nu}$ and mixed dark matter picture of the universe) and will be testable once the planned solar neutrino as well as $\beta \beta_{0 \nu}$ decay are carried out. To the extent that such an approach to understanding all available data using only three Majorana neutrinos was not discussed in literature, this work should be both of theoretical and experimental interest. It is also worth pointing out here that there are early results from the LSND experiment [17], which seem to indicate the oscillation of $\nu_{\mu}$ to $\nu_{e}$ with a $\Delta_{e \mu} \simeq 6 e V^{2}$. Should this result be confirmed after further data taking, all the scenarios discussed in this paper will be ruled out. Finally, of all the above scenarios, the one with all three neutrinos maximally mixed is an extremely symmetric possibility. We would therefore like to see if it can be derived in a plausible extension of the standard model.

III. Possible theoretical understanding of the maximally mixed degenerate neutrinos:

Let us now explore possible theoretical schemes for generating the maximally mixed three generation scenario, which, among all three scenarios described above may have the best chance of arising from some underlying symmetry. While we have not succeeded in finding a gauge theory where the above scheme can be generated in a technically natural manner, we have found a symmetry that such a theory ought to have in order to lead both to an almost degenerate mass pattern for neutrinos and maximal CP-violating mixing.

We consider the following extension of the standard model where the fermion sector is augmented by the addition of the three right-handed neutrinos ( denoted by $\nu_{R i}$ where $i$ is the generation index). Let us only focus on the lepton sector and denote the $S U(2)_{L}$ doublets by $\psi_{L i}$ and the right-handed singlets by $\ell_{i R}$. Let us assume that the theory prior to spontaneous symmetry breaking is invariant under a $Z_{3}$ symmetry,which has three elements $\left(1, S, S^{2}\right)$ ( i.e. $S^{3}=1$ ). Under the action of $S$, we have $\psi_{1 L} \rightarrow \psi_{2 L} ; \psi_{2 L} \rightarrow \psi_{3 L}$ and $\psi_{3 L} \rightarrow \psi_{1 L}$. So in generation space, we can write $S$ as a matrix:

$$
S=\left(\begin{array}{lll}
0 & 1 & 0 \\
0 & 0 & 1 \\
1 & 0 & 0
\end{array}\right)
$$

As for the remaining fermion fields, we assume that under the $Z_{3}, \tau_{R} \rightarrow \tau_{R}$; $\mu_{R} \rightarrow \omega^{2} \mu_{R}$ and $e_{R} \rightarrow \omega e_{R}$. If we have a Higgs doublet $H$ which is invariant under this $Z_{3}$ symmetry, then the following Yukawa coupling between fermions and the 
Higgs bosons is both gauge and $Z_{3}$ invariant:

$$
L_{1}=h_{0} \bar{\Psi}_{0 L} H \tau_{R}+h_{1} \bar{\Psi}_{1 L} H \mu_{R}+h_{2} \bar{\Psi}_{2 L} H e_{R}+\text { h.c. }
$$

where $\Psi_{0 L}=\frac{1}{\sqrt{3}}\left(\psi_{1 L}+\psi_{2 L}+\psi_{3 L}\right) ; \Psi_{1 L}=\frac{1}{\sqrt{3}}\left(\psi_{1 L}+\omega \psi_{2 L}+\omega^{2} \psi_{3 L}\right)$ and $\Psi_{2 L}=$ $\frac{1}{\sqrt{3}}\left(\psi_{1 L}+\omega^{2} \psi_{2 L}+\omega \psi_{3 L}\right)$. We add to this theory a Higgs triplet $\Delta_{L}$ with lepton number 2 which has a small vev which can be generated by see-saw mechanism for vev's a la ref. [6] by including a gauge singlet complex higgs $\Delta_{R}$ which has a large vev and is responsible for the right-handed Majorana neutrino mass in the see-saw matrix of eq.3. If the $\Delta_{L}$ is assumed to be a $Z_{3}$ singlet, then there are three gauge and $Z_{3}$ invariant couplings possible in the model: $\psi_{L}^{T} \psi_{L} \Delta_{L}, \psi_{L}^{T} S \psi_{L} \Delta_{L}$ and $\psi_{L}^{T} S^{2} \psi_{L} \Delta_{L}$ where we have assumed that $\psi_{L}^{T}=\left(\psi_{1 L}, \psi_{2 L}, \psi_{3 L}\right)$ is a row vector. In order to have a degenerate scenario, we will keep only the first invariant as part of our Yukawa Lagrangian :

$$
L_{2}=f_{L L} \psi_{L}^{T} \psi_{L} \Delta_{L}+\text { h.c. }
$$

As indicated after the $\Delta_{L}$ has a vev, it will generate a common Majorana mass for the light neutrinos. Finally, we will include in the theory three more Higgs doublets, which we denote by $\phi_{i}$ with $i=1,2,3$ such that they transform under the the $Z_{3}$ symmetry exactly as three lepton doublets. If we further assume the right handed neutrinos to be singlets under the $Z_{3}$, then there are several couplings allowed but we keep only a subset of those terms:

$$
L_{3}=\bar{\psi}_{1 L} \phi_{1} \nu_{1 R}+\bar{\psi}_{2 L} \phi_{2} \nu_{2 R}+\bar{\psi}_{3 L} \phi_{3} \nu_{3 R}+\Sigma_{i}\left(f_{i} \nu_{i R} \nu_{i R} \Delta_{R}\right)+\text { h.c. }
$$

After symmetry breaking $\left\langle H^{0}\right\rangle \neq 0,\left\langle\phi_{i}^{0}\right\rangle \neq 0$ as well as vev's for $\Delta_{L, R}^{0}$, one has a see-saw matrix for the neutrino sector, where there is no generation mixing and one has the modified see-saw matrix of eq.3 that leads to eq. 4 for the $\nu_{i}$ masses. The mixings arise purely from the charged lepton sector and it is easily seen that it has the desired maximal form as in eq.(5). We wish to emphasize again that we have not provided a technically natural derivation of the maximal mixing in the strict field theoretical sense but shown that any theory for such a mass and mixing pattern ought to have the generational $Z_{3}$ symmetry given in eq.(9). We envision this as arising from some high scale horizontal $S O(3)$ or $U(3)$ symmetry which after breakdown leaves the above $Z_{3}$ symmetry at low energies.

In conclusion, we have shown how a maximal CP-violating mixing among the three or two light Majorana neutrinos has the potential to accomodate the hot dark matter neutrino and the $\beta \beta_{0 \nu}$ data without at the same time contradicting the neutrino vacuum oscillation solutions to the solar and atmospheric neutrino data. 
While a detailed fit to the solar neutrino data discussed by other authors for similar scenarios is not completely satisfactory, at the present state of things, we are not discouraged by it. Clearly as the experimental situation is further sharpened, the fate of these models will be completely decided. We have also attempted to derive the maximal mixing pattern in extensions of the standard model.

\section{References}

[1] M. Gell-Mann, P. Ramond and R. Slansky, Supergravity, ed. D. Freedman et al, (North Holland, 1979); T. Yanagida, Proceedings of the conference of the baryon Number of the Universe, ed. (1979); R. N. Mohapatra and G. Senjanović, Phys. Rev. Lett. 44, 912 (1980).

[2] D. Chang, R. N. Mohapatra, J. Gipson, R. E. Marshak and M. K. Parida, Phys. Rev. D31, 1718 (1985); N. G. Deshpande, E. Keith and P. Pal, Phys. Rev. D46, 2261 (1992).

[3] CHORUS collaboration preprint CERN-SPSC/90-42 (1992); NOMAD collaboration preprint CERN-SPLSC/91-21 (1992).

[4] D. Caldwell and R. N. Mohapatra, Phys. Rev. D48, 3259 (1993) and ibid D50, 3477 (1994).

[5] W. Frati et al. Phys. Rev. D 48, 1140 (1993).

[6] R. N. Mohapatra and G. Senjanović, Phys. Rev. Lett. 44, 912 (1980); Phys. Rev. D23, 165 (1981).

[7] D. Caldwell and R. N. Mohapatra, ref[4]; D. G. Lee and R. N. Mohapatra, Phys. Lett. B 329, 463 (1994); S. T. Petcov and A. Smirnov, SISSA preprint 113/93/EP (1993); P. Bamert and C. Burgess, Phys. Lett.B ( to appear) (1994); A. Joshipura, PRL preprint (1993); A. Ioanyssian and J. W. F. Valle, Phys. Lett. B 332, 93 (1994); C. Albright and S. Nandi, Fermilab Preprint (1994); K. S. Babu and S. Pakvasa, Phys. Lett. B172, 360 (1986).

[8] For recent reviews, see H. Klapdor-Kleingrothaus, Prog. in Part. and Nucl. Physics 32, 261 (1994); D. Caldwell et al., Nucl. Phys. (Proc. Supp.) B13, 547, (1990); M. Moe and P. Vogel, Ann. Rev. of Nucl. and Part. Sc. ( to appear) (1994). 
[9] H. V. Klapdor-Kleingrothaus et al, Proceedings of the 27th International Conference on High Energy Physics, Glasgow (1994) (to appear); IGEX collaboration also reports an upper limit on $m_{\nu_{e}}$ of order $1 \mathrm{eV}$ ( private communication to S. Nussinov).

[10] S. Nussinov, Phys. Lett. 63B, 201 (1976).

[11] L. Wolfenstein, Phys. Rev. D 18, 958 (1978).

[12] NEMO 3 proposal, LAL 94-29 (1994).

[13] P. Krastev and A. Smirnov, Phys. Lett.B ( to appear). N. Hata and P. Langacker, Pennsylvania preprint (1994).

[14] R. Davis, Proceedings of the $6^{\text {th }}$ International Conference on Neutrino Telescopes, ed. M. Baldo-ceolin (to appear) (1994); Y. Suzuki, ibid.; Gallex Collaboration, Phys. Lett. B 327, 377 ( 1994); SAGE Collaboration, in Proceedings of the $27^{\text {th }}$ International Conference on High Energy Physics, Glasgow, July (1994); For reviews, see T. Kirsten, Proceedings of the International Conference on Non-Accelerator Physics, Bangalore, India, ed. R. Cowsik et al. ( to appear) (1994); T. Bowles, ibid.

[15] C. W. Kim and J. A. Lee, Johns Hopkins preprint, (1993); C. Giunti, C. W. Kim and J. D. Kim, SNUTH-94-109 (1994).

[16] P. Krastev and S. T. Petcov,Phys. Rev. Lett. 72, 1960 (1994); N. Hata, UPR0605T, (1994); Z. Berezhiani and A. Rossi, INFN FE-11-94.

[17] W. Louis, in XVI Conf. on Neutrino Phys. and Astrophys., Eilat, Israel (1994). 